\title{
Bronchoscopic radioisotope injection for sentinel lymph-node mapping in potentially resectable non-small-cell lung cancer ${ }^{\text {th }}$
}

\author{
D. Lardinois ${ }^{\mathrm{a}, *}$, T. Brack ${ }^{\mathrm{b}}$, A. Gaspert ${ }^{\mathrm{c}}$, T. Spahr ${ }^{\mathrm{d}}$, D. Schneiter ${ }^{\mathrm{a}}$, H.C. Steinert ${ }^{\mathrm{e}}$, W. Weder ${ }^{\mathrm{a}}$ \\ ${ }^{a}$ Division of Thoracic Surgery, University Hospital, Zurich, Switzerland \\ ${ }^{\mathrm{b}}$ Division of Pneumology, University Hospital, Zurich, Switzerland \\ ${ }^{\mathrm{c}}$ Department of Pathology, University Hospital, Zurich, Switzerland \\ ${ }^{\mathrm{d}}$ Department of Anaesthesiology, University Hospital, Zurich, Switzerland \\ ${ }^{\mathrm{e}}$ Division of Nuclear Medicine, University Hospital, Zurich, Switzerland
}

Received 24 October 2002; received in revised form 19 December 2002; accepted 27 January 2003

\begin{abstract}
Objective: Prospective study to evaluate the feasibility of a preoperative bronchoscopic radioisotope application, followed by conventional sentinel lymph-node (SLN) identification and to investigate the occurrence and distribution of micrometastases in relation to SLN activity. Methods: Twenty patients with a mean age of 63 years and proven clinical stage T1-3 N0-1 non-small-cell lung cancer (NSCLC) were included. A dosage of $80 \mathrm{MBq}$ radiolabeled technetium-99m nanocolloid was endoscopically administrated on intubated patients in the operation theatre. At thoracotomy, scintigraphic readings of both the primary tumor and hilar and mediastinal lymph-node stations were obtained with a hand-held gamma-counter. Patients underwent lung resection and mediastinal lymphadenectomy. Radiolabeled nodes were also examined separately on back-table. SLNs were defined as the hottest nodes or nodes with at least one-tenth of the radioactivity of the hottest nodes. SLNs pathologic assessment included standard examination using hematoxylin and eosin staining on step sections and immunohistochemistry (ICH) for cytokeratins. Results: Identification of SLNs was possible in 19/20 (95\%) patients after bronchoscopic radioisotope application. In 7/19 (37\%) patients, a unique SLN was identified, whereas in 12/19 (63\%) patients, nodes from two different stations could be classified as SLNs. Metastatic nodal disease was found in 9/19 (47\%) patients. ICH revealed micrometastases in 2/12 (17\%) patients, initially classified nodal negative. Pathologic negative SLNs were a predictor for absence of metastatic nodal disease after mediastinal lymphadenectomy. No complication related to the procedure was observed. Conclusion: Our preliminary results suggest that preoperative bronchoscopic radioisotope injection for SLN identification is a safe and simple method, improving accuracy of SLN detection in comparison to intraoperative technique. The absence of metastases in the SLNs seems to predict a negative nodal status accurately.
\end{abstract}

(C) 2003 Elsevier Science B.V. All rights reserved.

Keywords: Non-small-cell lung cancer; Sentinel lymph-node identification; Bronchoscopic technique; Radionucleide; Micrometastases

\section{Introduction}

Nodal status is admitted as the most significant predictor of survival for patients with non-small-cell lung cancer (NSCLC) [1,2]. As a consequence, adequate lymph-node staging is mandatory. Proper lymph-node staging is also required in patients with early stage NSCLC, since the prognostic significance of pathologic N1 seems to be

\footnotetext{
Presented at the 10th Annual Meeting of the European Society of Thoracic Surgeons, Istanbul, Turkey, October 26-28, 2002.

* Corresponding author. Tel.: +41-1-255-8802; fax: +41-1-255-8805.

E-mail address: didier.lardinois@chi.usz.ch (D. Lardinois).
}

different between intralobar and hilar involvement and since occult lymph-node metastases have been seen in up to $20 \%$ of the T1 and T2 tumors [3,4]. Positron emission tomography (PET) was demonstrated to be more sensitive and specific than a computed tomographic (CT) scan for mediastinal lymph-node assessment in patients with potentially operable NSCLC with a high negative predictive value [5]. However, micrometastases and especially microscopic nodal disease might be underestimated by PET due to its limitations to detect lesions smaller than $5 \mathrm{~mm} \mathrm{[6].}$ Improvement in the pathological examination due to the introduction of immunohistochemical staining lead to the identification of micrometastases in $15 \%$ of the histologi- 
cally node negative NSCLC [7]. Since the 5-year survival rate $(62 \%)$ of patients with micrometastases seemed to be significantly lower than that of patients without micrometastases $(86 \%, P=0.004)$ in patients with peripheral lung adenocarcinoma of $2.0 \mathrm{~cm}$ or less in diameter, adjuvant therapy might be helpful in the patients with nodal micrometastases [4]. In this respect, the sentinel lymphnode (SLN) technique might increase the accuracy of surgical lymph-node staging and assess the prognostic significance of micrometastatic lymph-node involvement in patients with early stage NSCLC. SLN mapping with bluedye technique and with radioisotopic technetium (Tc)-99m has been shown as a safe way of identifying the first site of potential nodal metastases in up to $85 \%$ of the lung cancer patients $[1,2,7,8]$. Micrometastases could be found in about $20-30 \%$ of the positive SLN [7]. The investigators used an intraoperative or a preoperative CT-guided injection technique for these questions. Using the intraoperative technique, blue-dye or radioisotope injection was performed on atelectatic lung and waiting time, until migration of the substance was achieved, could be long [2]. In this prospective study, the main objective was to evaluate the feasibility of a new preoperative bronchoscopic radioisotope application, followed by conventional SLN identification technique. Additionally, the occurrence and distribution of micrometastases in relation to SLN activity was investigated.

\section{Material and methods}

\subsection{Patients}

Twenty patients with proven NSCLC were prospectively included in this study between January and September 2002. The cohort consisted of 14 men and six women, with a mean age of 63.5 years (range 48-82 years). The protocol was approved by the Ethic Committee and the Review Board for protocols dealing with human subjects at the University of Zurich and the patients were enrolled after informed consent. Conventional staging by means of a history, physical findings, blood test, bronchoscopy and contrastmaterial-enhanced CT scan of the chest and upper abdomen was performed in all patients. All patients underwent integrated whole-body PET/CT for mediastinal staging and to exclude distant metastases. No patient had suspicion of mediastinal nodal involvement at the preoperative integrated PET/CT. In all patients, the clinical tumor stage was T1-3 N0-1 and surgical treatment was intended.

\subsection{Preoperative endoscopic application of radioisotope}

Patients were initially intubated with a single-lumen endotracheal tube. Then, bronchoscopy with a fiberoptic endoscope was performed through the endotracheal tube. When the tumor was bronchoscopically visible, a protected needle (MAJ 64/65, 21 gauge $\times 13 \mathrm{~mm}$, Olympus Optical Corporation, Japan) was inserted through the endoscope and the needle-tip was transbronchially inserted at the tumor margin. When the tumor was not visible endobronchially, the needle was inserted at the carina of the most distal pulmonary sub-segment that could be reached endoscopically in the proximity of the tumor according to its location on preoperative CT scan. A mean dosage of $80.2 \mathrm{MBq}$ (2 mCi, range 78.3-82.6 MBq) radiolabeled Tc-99m nanocolloid in $1 \mathrm{ml}$ was injected. After injection, a double-lumen tubus was introduced and the patient was positioned for lung resection. The mean duration of the bronchoscopic procedure including the transbronchial injection of Tc was 4 min (range 2-12 $\mathrm{min}$ ).

\subsection{Intraoperative identification of the sentinel lymph- node( $(s)$}

After anterolateral thoracotomy, the value of counts per second of the primary tumor and intrathoracic nodal stations were taken with a hand-held gamma-probe counter $35^{\circ}$ (Navigator system, Tyco Healthcare Switzerland, 8832 Wollerau). Lung resections were performed with formal mediastinal lymph-node dissection (MLND) according to the American Thoracic Society (ATS) mapping system [9]. Measurement of radioactivity was repeated in all the lymphnode stations and in the tumor specimen on back-table. The nodes which were considered as sentinel nodes consisted of the hottest nodes and additionally, all the nodes containing more than one-tenth of the activity of the hottest nodes were removed.

\subsection{Pathologic evaluation}

The hottest sentinel nodes were formalin fixed and paraffin embedded. They were examined on three-step sections taken at $250 \mu \mathrm{m}$ using hematoxylin and eosin (HE) staining. If no metastases were found, additional immunohistochemistry (IHC) for cytokeratins was performed on unstained slides prepared with the step sections. The additional sentinel nodes with a radioactivity greater than one-tenth of the hottest nodes were examined with three additional step sections and IHC. IHC was evaluated as positive if demonstrating individual cells or cell clusters consistent with metastastic cells of the respective carcinoma.

\section{Results}

Identification of SLNs was possible in 19/20 (95\%) patients after preoperative bronchoscopic injection of Tc$99 \mathrm{~m}$. In one patient, no radioactivity could be measured at thoracotomy, $1 \mathrm{~h}$ after administration of Tc-99m. This patient suffered from a squamous cell carcinoma localized in the apical lower-lobe segment and no technical difficulty 
was observed during injection of Tc-99m. The mean-time interval between endoscopic injection and first measurement of radioactivity with a hand-held gamma-probe was $1.4 \mathrm{~h}$ (range $1-3 \mathrm{~h}$ ). The mean-time interval between injection of Tc-99m and definitive identification of SLN on back-table was $3.3 \mathrm{~h}$ (range 2-4.5 h). In 7/19 (37\%) patients, a unique sentinel node was identified, whereas in $12 / 19$ (63\%), two separate stations could be classified as sentinel nodes. These two stations corresponded to the hottest nodes and to the nodes with radioactive counts $\geq$ one-tenth of the hottest nodes. The hottest SLN were localized in a N1-station in 15/19 (79\%) patients, including ATS 10, 11, 12, 13 in three, six, five and one patients, respectively. Mediastinal N2 localization was subcarinal (ATS 7) in three patients and in the aorto-pulmonary window (ATS 5) in one. No adverse effects related to the procedure were observed.

\subsection{Detection of metastases}

Metastatic disease was found in 9/19 (47\%) of the patients within the identified SLNs. Metastases was found in a single SLN in $7 / 9(78 \%)$ of these patients and simultaneously in two SLNs in $2 / 9$ patients $(22 \%)$. In the seven patients with single nodal metastases, the hottest sentinel node was involved in 5/7 (71\%); in 2/7 (29\%), metastases were localized in a less radioactive sentinel node, while the most radioactive node was negative for tumor. In all seven patients with single SLN metastases, the involved node stations were N1. In two patients, a multilevel nodal involvement was observed. The hottest positive SLN was localized in a N1-station, but metastases were also found in a less radioactive ( $>$ one-tenth) N2 station. In 4/19 (21\%) of patients, SLN was localized in the mediastinum (N2), showing a skip-pattern drainage by radionucleide activity, but no metastases could be found either by conventional histopathologic evaluation or by ICH.

In 2/9 (22\%) of patients with nodal metastases, the SLN was negative by conventional histopathologic evaluation. Micrometastases and clusters of tumor cells were only identified after serial sectioning of the nodal tissue and ICH evaluation by cytokeratin antibody staining in these two patients. Micrometastases were identified in a N1-lymphnode in both patient, one in the hottest node and one in a less radioactive (>one-tenth) SLN. These two patients were upstaged by the SLN procedure with a stage migration from stage T2N0 IB to stage T2N1 IIB.

\subsection{Accuracy of the technique}

In the ten patients with no tumor found in the SLN, there was also no metastatic disease even after extended mediastinal lymphadenectomy in nine of them. In one of these patients with negative SLN in position ATS 12, three metastatic intrapulmonary nodes were observed in position
ATS 13. However, these involved nodes were encased in the centrally located tumor (squamous cell carcinoma) and could not be macroscopically separated any more. Thus, pathologic negative SLNs were a predictor for absence of metastatic nodal disease after MLND in this series.

In nine patients, metastatic disease in the SLN was the only site even after MLND. The hottest SLN was the only positive node in 6/9 of these patients. In two patients with tumor positive hottest N1 SLN, metastatic disease was also found in a mediastinal N2 lymph-node station. However, these two N2 lymph-nodes had a radioactivity $\geq$ one-tenth of the hottest node and could be considered as separate sentinel nodes.

\section{Discussion}

\subsection{Preoperative bronchoscopic technique}

The bronchoscopic injection of the radionucleide allowed a SLN identification in $95 \%$ of patients. Preoperative CT-guided injection of Tc-99m in patients with NSCLC for identification of SLN was already shown as a reliable method with a SLN localization in $87 \%$ of the patients [8]. These results seem to be superior to the intraoperative technique where a sentinel node could be found in $47-82 \%$ of patients $[1,2]$. It may be due to a more physiological way of injecting Tc-99m on ventilated lungs and to the fact that the migration of the radionucleide was not affected during preparation of the structures to be resected as with the intraoperative technique. The bronchoscopic technique might provide an advantage, being less invasive for the patient than the transthoracic approach and revealing no complications in our experience. Contrary to previous reports [2], we did not observe failure of radionucleide migration in large tumors with central necrosis.

\subsection{Definition of $S L N$}

In a previous report dealing with melanoma, a less radioactive node was found to be positive for metastatic disease in $13 \%$, despite the most radioactive node being negative $[10,11]$. Therefore, the authors recommended to consider as SLNs, not only the hottest nodes but also the nodes containing at least one-tenth or more of the ex vivo count of the hottest sentinel node [10]. In our experience, 2/ 13 patients (15\%) with negative hottest SLN showed metastases in a less radioactive node, which correlates with the findings in melanoma and breast cancer studies and supports our choice for definition of SLN [11].

\subsection{Accuracy of the bronchoscopic technique}

In $9 / 10$ patients, the negative sentinel nodes represented the final N0 stage after MLND. These results corroborate the findings of others, using pre- or intraoperative injection 
of the radionucleide [1,12]. In a patient with negative peribronchial SLN, three intrapulmonary nodes encased by the tumor were found to be positive. However, we consider this not a failure since these nodes were adjacent to the tumor. Therefore, these results suggest that a pathologic negative SLN reflects a node negative mediastinum accurately. Furthermore, all nodal metastases were found in SLNs, either in the hottest nodes, or in less radioactive SLNs. Radioactive nodes with activity below the threshold of one-tenth of the hottest were negative in all cases. However, despite the excellent specificity of the bronchoscopic technique, these results have to be considered carefully, due to the small number of patients included in this study. Therefore, the question if patients with NO SLNs might be spared more extensive lymph-node dissection cannot be clearly answered at this time of investigation. In order to spare extensive mediastinal lymphadenectomy, frozen-section examination of the SLNs should be available. However, frozen-section analysis was demonstrated to be less sensitive in detecting micrometastatic disease with 54\% of correct identification of a positive SLN in patients with breast cancer [12-14]. A serial sectioning examination with HE section per 2-3 mm-thick block of lymph-node and routine ICH seem to remain the golden standard to find micrometastases [15].

\subsection{Micrometastases}

The presence of micrometastatic disease in lymph-nodes might be of high prognostic relevance, since previous reports for colo-rectal carcinoma and NSCLC mentioned survival curves significantly worse [16,17]. The patients with micrometastatic disease, only found in the SLN in our series, represented $17 \%(2 / 12)$ of those initially classified nodal negative by standard histologic examination and $11 \%$ of the 19 patients with identified SLN. Thus, in nearly $1 / 10$ patients, staging was directly affected, which confirmed the results of Liptay et al. who found micrometastases in seven of 91 patients with NSCLC [7]. It has been shown that allowing the pathologists to focus on either a single lymphnode or relatively few nodes improves their results in finding micrometastases [18].

In conclusion, our results suggest that SLN mapping by use of a preoperative bronchoscopic radioisotope technique was easy to perform and safe. The diagnostic yield in the identification of SLN was greater than by the intraoperative technique. Furthermore, SLN analysis seems to predict a pathologic N0 staging status accurately when no metastases were present in the sentinel node. It allows the pathologist to apply more sensitive but time-consuming techniques selectively to assess occult lymphatic metastases. In this respect, SLN technique might refine the nodal staging and allow us to gain better insight into the biologic behavior of subgroups of NSCLC which may have an impact on adjuvant therapies in the future.

\section{References}

[1] Little AG, DeHoyos A, Kirgan DM, Arcomano TR, Murray KD. Intraoperative lymphatic mapping for non-small cell lung cancer: the sentinel node technique. J Thorac Cardiovasc Surg 1999;117:220-4.

[2] Liptay MJ, Masters GA, Winchester DJ, Edelman BL, Garrido BJ, Hirschtritt TR, Perlman RM, Fry WA. Intraoperative radioisotope sentinel lymph node mapping in non-small cell lung cancer. Ann Thorac Surg 2000;70:384-90.

[3] Asamura H, Suzuki K, Kondo H. Where is the boundary between N1 and N2 stations in lung cancer? Ann Thorac Surg 2000;70:1839-45.

[4] Wu J, Ohta Y, Minato H, Tsunezuka Y, Oda M, Watanabe Y, Watanabe G. Nodal occult metastasis in patients with peripheral lung adenocarcinoma of $2.0 \mathrm{~cm}$ or less in diameter. Ann Thorac Surg 2001; 71:1772-8.

[5] Pieterman RM, van Putten JWG, Meuzelaar JJ, Mooyaart EL, Vaalburg W, Koeter GH, Fidler V, Pruim J, Groen HJM. Preoperative staging of non-small cell lung cancer with positron-emission tomography. N Engl J Med 2000;343:254-61.

[6] Kernstine KH, McLaughlin KA, Menda Y, Rossi NP, Kahn DJ, Bushnell DL, Graham MM, Brown CK, Madsen MT. Can FDG-PET reduce the need for mediastinoscopy in potentially resectable nonsmall cell lung cancer? Ann Thorac Surg 2002;73:394-402.

[7] Liptay MJ, Grondin SC, Fry WA, Pozdol C, Watkin W. Intraoperative sentinel lymph node mapping in non-small-cell lung cancer improves detection of micrometastases. J Clin Oncol 2002;20:1984-8.

[8] Nomori H, Horio H, Naruke T, Orikasa H, Yamazaki K, Suemasu K. Use of technetium-99m tin colloid for sentinel lymph node identification in non-small cell lung cancer. J Thorac Cardiovasc Surg 2002;124:486-92.

[9] American Thoracic Society. Clinical staging of primary lung cancer. Am Rev Respir Dis 1983;127:1-6.

[10] Coit DG. The 'true' sentinel lymph node: in search of an operational definition of a biological phenomenon. Ann Surg Oncol 2001;8: 187-9.

[11] McMasters KM, Reintgen DS, Ross MI, Wong SL, Gershenwald JE, Edwards MJ. Sentinel lymph node biopsy for melanoma: how man radioactives nodes should be removed? Ann Surg Oncol 2001;8: $192-7$.

[12] Schmidt FE, Woltering EA, Webb WR, Garcia OM, Cohen JE, Rozans MH. Sentinel nodal assessment in patients with carcinoma of the lung. Ann Thorac Surg 2002;74:870-5.

[13] Kane JM, Edge SB, Winston JS, Watroba N, Hurd TC. Intraoperative pathologic evaluation of a breast cancer sentinel lymph node biopsy as a determinant for synchronous axillary lymph node dissection. Ann Surg Oncol 2001;8:361-7.

[14] Tanis PJ, Boom RPA, Koops HS. Frozen section investigation of the sentinel node in malignant melanoma and breast cancer. Ann Surg Oncol 2001;8:222-6.

[15] McCready DR. Intraoperative sentinel node assessment is a touchy subject. Ann Surg Oncol 2002;9:321-3.

[16] Kubuschock B, Passlick B, Izbicki JR. Disseminated tumor cells in lymph nodes as a determinant for survival in surgically resected nonsmall cell lung cancer. J Clin Oncol 1999;17:19-24.

[17] Perez-Cardona JH, Ordonez NG, Fossella FV. Lymph node micrometastases in non-small cell lung cancer: clinical applications. Clin Lung Cancer 2000;2:116-20.

[18] Liptay MJ. Commentary on sentinel lymph node identification with technetium-99m tin colloid in non-small cell lung cancer. J Thorac Cardiovasc Surg 2002;124:428-30. 